\title{
Early diagnosis and treatment of gastrointestinal neuroendocrine tumors
}

\author{
HONG SHEN ${ }^{1}$, ZHUO YU ${ }^{1}$, JING ZHAO ${ }^{1}$, XIU-ZHEN LI $^{2}$ and WEN-SHENG PAN ${ }^{3}$ \\ Departments of ${ }^{1}$ Medical Oncology, ${ }^{2}$ Pathology and ${ }^{3}$ Gastroenterology, \\ The Second Affiliated Hospital, Zhejiang University School of Medicine, Hangzhou, Zhejiang 310009, P.R. China
}

Received January 25, 2015; Accepted February 24, 2016

DOI: $10.3892 / 01.2016 .5062$

\begin{abstract}
The aim of the present retrospective analysis on the macroscopic appearance and pathological characteristics of gastrointestinal neuroendocrine tumors (NETs) was to investigate methods for their early diagnosis and treatment. A total of 78 patients were divided into submucosal NET and deeper invasion NET groups, according to the depth of tumor invasion exhibited by the patients. The clinicopathological characteristics and survival time of the NET patients were analyzed and compared. The pathological characteristics of the submucosal NETs group were investigated according to the diameter of the tumor $(\leq 5.0 \mathrm{~mm}, 5.1-10.0 \mathrm{~mm}$ or $>10.0 \mathrm{~mm}$ ). The depth of invasion at diagnosis was observed to significantly correlate with histopathological classification, diameter of the tumor, macroscopic appearance, growth pattern, lymphatic-vascular involvement, lymph node (LN) metastasis and distant metastasis. In the submucosal NETs group, high-grade tumors with lymphatic or venule invasion and distant metastasis were associated with an increased risk of nodal metastases. In patients with minute tumors $(\leq 5.0 \mathrm{~mm})$, no lymphatic-vascular involvement, LN or distant metastasis was observed. By contrast, patients with tumors measuring 5.1-10.0 $\mathrm{mm}$ in diameter exhibited high lymphatic-vascular involvement and LN metastasis rates (46.2 and 30.8\%, respectively). Survival time was significantly longer in patients with submucosal NETs compared with deeper invasion NETs and in patients with NET G1 and NET G2 compared with NEC. The results of the present study indicate that gastrointestinal submucosal NETs are closely associated with a slightly elevated macroscopic type, low-grade tumors and a small diameter. These features may contribute to early diagnosis of gastrointestinal NETs. Therefore, a tumor diameter of
\end{abstract}

Correspondence to: Professor Wen-Sheng Pan, Department of Gastroenterology, The Second Affiliated Hospital, Zhejiang University School of Medicine, 88 Jie-Fang Road, Hangzhou, Zhejiang 310009, P.R. China

E-mail: wspan223@163.com

Key words: neuroendocrine tumors, gastrointestinal tract, early diagnosis, treatment, endoscopy
$<5.0 \mathrm{~mm}$, with slightly elevated macroscopic appearance may indicate an absolute requirement for endoscopic resection, while tumors measuring $5.1-10.0 \mathrm{~mm}$ in diameter must be considered carefully.

\section{Introduction}

Neuroendocrine tumors (NETs) were previously considered extremely rare lesions, which most commonly occur in the gastroenteropancreatic system $(1,2)$. However, over the last 30 years, the incidence and prevalence of gastrointestinal NETs have increased significantly due to increased awareness and widespread use of gastrointestinal endoscopy $(3,4)$. Gastroenteropancreatic NETs, which derive from the neuroendocrine cell system and have widely divergent clinical presentations, are relatively infrequent, constituting 2\% of all neoplasms, and are typically indolent, slow-growing tumors $(5,6)$. Gastrointestinal NETs are increasing (7); in the USA, the prevalence and the incidence of gastrointestinal NETs have been calculated to be 35/100,000 and 5/100,000, respectively (4), revealing a 7 -fold increase in the last 35 years. This phenomenon may partly reflect the increased number of diagnoses of benign and incidentally-identified lesions due to the increased availability of advanced endoscopic and radiological imaging (8-10). The overall 5-year survival rate for patients with gastrointestinal NETs has improved by almost $20 \%$ in the past 35 years $(1,11,12)$. Diagnosis of early-stage NETs remains difficult, and the majority of patients exhibit locally advanced disease or distant metastasis at diagnosis (4).

At present, an increasing number of gastrointestinal NETs are diagnosed at an early stage, and thus may be treated with local excision (13). According to the National Comprehensive Cancer Network guidelines and American Joint Committee on Cancer staging of gastrointestinal NETs (14), T1 tumors that are limited to the lamina propria or submucosa and present a diameter of $<1 \mathrm{~cm}$ (the diameter of colorectal and appendix $\leq 2 \mathrm{~cm}$ ), are considered appropriate for endoscopic resection $(15,16)$. However, previous studies have reported that patients with such tumors have experienced lymphatic-vascular involvement and exhibit the risk of recurrence and metastasis following endoscopic resection (13). In the present study, the clinical and pathological features of 78 gastrointestinal NET patients were analyzed to identify appropriate methods for early diagnosis and treatment of this type of tumors. 


\section{Materials and methods}

Patients. The medical records of 78 patients with histopathologically diagnosed gastrointestinal NETs that were treated at The Second Affiliated Hospital of Zhejiang University School of Medicine (Hangzhou, China) between January 2007 and March 2013 were retrospectively analyzed. The patient cohort included $42(53.8 \%)$ male patients and $36(46.2 \%)$ female patients with a mean age at diagnosis of 58 years (range, $15-82$ years) (Table I).

Patients were divided into two groups, namely, a submucosal NETs group and a deeper invasion NETs group, according to the depth of tumor invasion. The present study defined submucosal NETs as tumors that were restricted to the lamina propria or submucosal layers, with or without lymph node (LN) and distant organ metastasis. Deeper invasion NETs were defined as tumors that had infiltrated into the muscularis propria or deeper structures, with or without LN and distant organ metastasis. The pathological characteristics of the submucosal NETs group were investigated according to the tumor diameter $(\leq 5.0,5.1-10.0$ or $>10.0 \mathrm{~mm})$. A minute tumor was defined as a tumor of $\leq 5.0 \mathrm{~mm}$ in diameter.

The submucosal NETs group consisted of 24 patients (11 males and 13 females) with a mean age of 57 years (range, 29-70 years). The tumors in this group penetrated the mucosa and were restricted to the submucosa. The deeper invasion NETs group consisted of 54 patients ( 31 males and 23 females) with a mean age of 62 years (range, $15-82$ years).

Macroscopic appearance of tumors. According to the Paris classification of superficial neoplastic lesions $(17,18)$, early-stage gastric cancer is classified into three types: Slightly elevated type (type $0-\mathrm{I}$ ), flat type (type $0-\mathrm{II}$ ) and concaved type (type 0 -III). Endoscopy was performed using the GIF-XQ240, GIF-H260, GIF-H260Z, CF-240I/L, CF-H260AI and PCF-Q260AZI endoscopes (Olympus Corporation, Tokyo, Japan) Based on the endoscopic characteristics of the NETs, submucosal NETs were classified into two types: Elevated type (type 0-Is), which consists of elevating lesions such as submucosal tumors, and elevated depressed type (type 0-Is+IIc), which consists of an elevating lesion with a depressed lesion. Deeper invasion NETs were endoscopically classified into four types: Borrmann's type I, Borrmann's type II, Borrmann's type III type and Borrmann's type IV (19).

NET growth pattern. Based on the tumor growth pattern, gastrointestinal NETs were classified as exhibiting an expansive or infiltrative pattern. Certain NETs with an infiltrative growth pattern exhibited various invasion directions, including infiltration towards the anterior and lateral sides of the tumors.

Histopathological classification. All specimens were dissected at a maximal section. Hematoxylin (catalog no., 130608; Shanghai Boao Biotechnology Co., Ltd., Shanghai, China) and eosin (catalog no., 20130729; Shanghai SSS Reagent Co., Ltd., Shanghai, China) staining and immunohistochemical staining for synuclein- $\alpha$ (rabbit anti-human polyclonal antibody; cat. no. ZM-0506; dilution,
1:300), chromogranin A (EP38; rabbit anti-human polyclonal antibody; cat. no. ZA-0507; dilution, 1:200), neuron-specific enolase (E27; mouse anti-human polyclonal antibody; cat. no. ZM-0203; dilution, 1:200) and Ki-67 (MIB1; mouse anti-human polyclonal antibody; cat. no. ZM-0167; dilution, 1:300) (Beijing Zhongshan Golden Bridge Biotechnology Co., Ltd., Beijing, China) were subsequently performed using the One-Step Universal kit (containing endogenous peroxidase blocking solution, HRP-Polymer and DAB Diluent; catalog no., PV-8000; Beijing Zhongshan Golden Bridge Biotechnology Co., Ltd.). Antibodies were incubated for $32 \mathrm{~min}$ at $37^{\circ} \mathrm{C}$. Stained sections were examined using a Nikon ECLIPSE 50I microscope (Nikon Corporation, Tokyo, Japan). According to the 2010 World Health Organization International Classifications of Tumors (20), NETs were classified into four subtypes: NET G1, NET G2, neuroendocrine carcinoma (large or small cell carcinoma) and mixed adenoneuroendocrine carcinoma.

Statistical analysis. All statistical analyses were performed with SPSS version 20.0 statistical software (IBM SPSS, Armonk, NY, USA). The $\chi^{2}$ test was used to evaluate the associations between the depth of invasion and clinicopathological variables of the patients and the association between LN metastasis and clinicopathological variables. Survival was estimated according to the Kaplan-Meier product limit estimator, and differences observed among patient subgroups were assessed by the log-rank test. $\mathrm{P}<0.05$ was considered to indicate a statistically significant difference.

\section{Results}

Characteristics of gastrointestinal NET patients. A total of 78 patients with gastrointestinal NETs were included in the present study. The baseline characteristics of the patients are presented in detail in Table I. The median age of the patients was 58 years old. Out of 78 patients, $42(53.8 \%)$ were men and $36(46.2 \%)$ were women. The primary tumor sites were the esophagus $(11 / 78 ; 14.1 \%)$, the duodenum $(6 / 78 ; 7.7 \%)$, the stomach $(30 / 78 ; 38.5 \%)$, the colon $(6 / 78 ; 7.7 \%)$, the rectum $(23 / 78 ; 29.5 \%)$ and the appendix $(2 / 78 ; 2.6 \%)$. In total, 24 patients $(30.8 \%)$ possessed distant metastasis, while the remaining 54 (69.2\%) had no distant metastasis.

Association between patients' clinicopathological characteristics and depth of tumor invasion. The association between the clinicopathological characteristics of the patients and the depth of tumor invasion is summarized in Table II.

No significant differences were identified between age $(\mathrm{P}=0.409)$ and gender groups $(\mathrm{P}=0.350)$ in terms of depth of tumor invasion. However, a significant correlation was identified between depth of tumor invasion at diagnosis and histopathological classification $(\mathrm{P}<0.001)$, diameter of the tumor $(\mathrm{P}<0.001)$, macroscopic appearance $(\mathrm{P}<0.001)$, growth pattern $(\mathrm{P}<0.001)$, lymphatic-vascular involvement $(\mathrm{P}<0.001), \mathrm{LN}$ metastasis $(\mathrm{P}<0.001)$ and distant metastasis $(\mathrm{P}=0.001)$.

In the submucosal NETs group, 9 (37.5\%) patients exhibited an expansive growth pattern and $15(62.5 \%)$ exhibited an infiltrative growth pattern, of which 10 cases infiltrated towards the anterior side of the tumor, 2 cases infiltrated towards the lateral 
Table I. Characteristics of 78 patients with gastrointestinal neuroendocrine tumors.

\begin{tabular}{lc} 
Parameter & Patients, $\mathrm{n}(\%)$ \\
\hline Gender & \\
Male & $42(53.8)$ \\
Female & $36(46.2)$ \\
Mean age, years (range) & $58(15-82)$ \\
Primary tumor site & \\
Esophagus & $11(14.1)$ \\
Duodenum & $6(7.7)$ \\
Stomach & $30(38.5)$ \\
Colon & $6(7.7)$ \\
Rectum & $23(29.5)$ \\
Appendix & $2(2.6)$ \\
Distant metastasis & \\
pM0 & $54(69.2)$ \\
pM1 & $24(30.8)$ \\
\hline
\end{tabular}

side of the tumor and 3 cases infiltrated towards the anterior and lateral sides of the tumor. In the deeper invasion NETs group, $54(100.0 \%)$ cases exhibited an infiltrative growth pattern.

Association between patients' clinicopathological factors and LN metastasis in submucosal gastrointestinal NETs. The association between different clinical and pathological factors of submucosal NETs and the risk of LN metastasis is presented in Table III.

A significant correlation was identified between high-grade tumors with lymphatic or venule invasion or distant metastasis and an increased risk of nodal metastases $(\mathrm{P}<0.001, \mathrm{P}=0.027$ and $\mathrm{P}=0.049$, respectively).

In the submucosal NETs group, 7 minute tumors $(\leq 5.0 \mathrm{~mm})$ exhibited an expansive growth pattern ( 0 -Is type), with no evidence of lymphatic-vascular involvement, LN or distant metastasis. Tumors measuring 5.1-10.0 $\mathrm{mm}$ in diameter exhibited a high rate of infiltrative growth $(84.6 \%$; 11/13) (Figs. 1 and 2), lymphatic-vascular involvement (46.2\%; 6/13), LN metastasis $(30.8 \% ; 4 / 13)$ and distant metastasis $(7.7 \% ; 1 / 13)$. Tumors measuring $>10.0 \mathrm{~mm}$ in diameter also exhibited a high rate of lymphatic-vascular involvement $(75.0 \% ; 3 / 4)$ and $\mathrm{LN}$ metastasis $(25.0 \%$; 1/4). No significant difference in LN metastasis was identified between the three tumor diameter categories $(\mathrm{P}=0.238)$.

Therapy. Of the 24 submucosal NETs, 1 case (4.2\%) of liver metastasis was identified in a patient who had undergone resection of a primary tumor. In the other 23 cases, 14 cases $(60.9 \%)$ underwent endoscopic resection of the primary tumor and 5 cases $(21.7 \%)$ received surgery following endoscopic resection for suspected positive margin or lymphatic-vascular involvement. The other 9 cases (39.1\%) underwent surgery.

In the deeper invasion NETs group, 23 (42.6\%) patients with distant metastasis and $31(57.4 \%)$ patients without distant metastasis underwent surgical resection of the primary tumor. Among the 23 patients exhibiting distant metastasis, 14 underwent surgical resection of the primary tumor: 4 cases $(17.4 \%)$ underwent resection of the metastatic disease and 10 cases (43.5\%) received systemic therapy, which included somatostatin analogues (Sandostatin LAR; $30 \mathrm{mg}$ on day 1, every 28 days; Novartis International AG, Basel, Switzerland) and chemotherapy $\left(100 \mathrm{mg} / \mathrm{m}^{2}\right.$ etoposide on days $1-3$ and $25 \mathrm{mg} / \mathrm{m}^{2}$ cisplatin on days $1-3$; every 21 days, for 4-6 cycles).

Follow-up. The mean follow-up period, post-surgery or treatment, was 40 months. In total, $12(15.4 \%)$ of the 78 patients succumbed to the disease during the follow-up period. All patients in the submucosal NETs group survived (follow-up time, 33 months). No evidence of recurrence or metastasis was identified in 23 (95.8\%) cases, whereas rectal NETs with liver metastasis was diagnosed in $1(4.2 \%)$ patient. This patient was a 29-year-old male, with an 8-mm primary tumor (type 0-Is; NET G2), which exhibited infiltrative growth, lymphatic-vascular involvement and was LN-positive. The patient had undergone resection of the primary tumor and no evidence of recurrence or disease progression was identified during the follow-up period. In the submucosal NETs group, the 3-year survival and disease-free survival rates were 100.0 and $95.8 \%$, respectively. In the deeper invasion NETs group, $12(22.2 \%)$ patients succumbed to the disease, and the 3 -year survival rate was $83.3 \%$.

A significant correlation was identified between submucosal NETs or lower grade tumors and prolonged overall survival $(\mathrm{P}<0.001$ and $\mathrm{P}=0.009$, respectively; Table IV). No significant differences were identified between age or gender and overall survival $(\mathrm{P}=0.276$ and $\mathrm{P}=0.089$, respectively).

\section{Discussion}

As the incidence of gastrointestinal NETs has increased, endoscopic therapy has become more common for the treatment of this type of tumors $(3,4)$. Therefore, studies investigating the safety, potential risks and indications of endoscopic therapy have become particularly important.

Several studies have demonstrated that the histological classification, grading, LN metastasis, lymphatic-vascular involvement and perineural invasion of gastrointestinal NETs are associated with the infiltrative growth of the tumor, staging and prognosis (4,21-23). For early-stage gastric cancer, tumors exhibiting an expansive growth pattern and elevated macroscopic type are less invasive, whereas poorly differentiated tumors are associated with a higher risk of deep infiltration, LN and distant metastasis $(24,25)$. In the present study, histologically submucosal NETs exhibited a higher level of differentiation, expansive growth and small diameter compared with deeper invasion NETs. In the deeper invasion NETs group, an infiltrative growth pattern and tumors of mixed and poorly differentiated type were more common than in the submucosal NETs group. These findings indicated that poorly differentiated NETs usually exhibit an infiltrative growth pattern and are more likely to invade the muscularis propria and deeper layer and exhibit LN and distal metastasis.

Thomas et al (26) analyzed 104 gastric carcinoids, the majority of which exhibited a diameter of $<2.0 \mathrm{~cm}$, and observed that the infiltration depth was restricted 
Table II. Association between clinicopathological characteristics and depth of tumor invasion in 78 gastrointestinal NET patients.

\begin{tabular}{|c|c|c|c|c|}
\hline Parameter & Patients, $\mathrm{n}$ & $\begin{array}{l}\text { Submucosal } \\
\text { NETs, n }(\%)\end{array}$ & $\begin{array}{c}\text { Deeper invasion } \\
\text { NETs, n }(\%)\end{array}$ & P-value \\
\hline Gender & & & & 0.350 \\
\hline Male & 42 & $11(45.8)$ & $31(57.4)$ & \\
\hline Female & 36 & $13(54.2)$ & $23(42.6)$ & \\
\hline Age, years & & & & 0.409 \\
\hline$>30$ & 6 & $1(4.2)$ & $5(9.3)$ & \\
\hline $31-60$ & 33 & $14(58.3)$ & $19(35.2)$ & \\
\hline$>60$ & 39 & $9(37.5)$ & $30(55.6)$ & \\
\hline Tumor location & & & & 0.026 \\
\hline Esophagus & 11 & $2(8.3)$ & $9(16.7)$ & \\
\hline Duodenum & 6 & $2(8.3)$ & $4(7.4)$ & \\
\hline Stomach & 30 & $6(25.0)$ & $24(44.4)$ & \\
\hline Colon & 6 & $0(0.0)$ & $6(11.1)$ & \\
\hline Rectum & 23 & $14(58.3)$ & $9(16.7)$ & \\
\hline Appendix & 2 & $0(0.0)$ & $2(3.7)$ & \\
\hline Histopathological classification & & & & $<0.001$ \\
\hline NET G1 & 20 & $20(83.3)$ & $0(0.0)$ & \\
\hline NET G2 & 29 & $3(12.5)$ & $26(48.1)$ & \\
\hline $\mathrm{NEC}$ & 9 & $0(0.0)$ & $9(16.7)$ & \\
\hline MANEC & 20 & $1(4.2)$ & $19(35.2)$ & \\
\hline Tumor diameter, mm & & & & $<0.001$ \\
\hline$\leq 10.0$ & 20 & $20(83.3)$ & $0(0.0)$ & \\
\hline$>10.0$ & 58 & $4(16.7)$ & $54(100.0)$ & \\
\hline Macroscopic appearance & & & & $<0.001$ \\
\hline 0 -Is type & 18 & $18(75.0)$ & $0(0.0)$ & \\
\hline 0-Is+IIc type & 6 & $6(25.0)$ & $0(0.0)$ & \\
\hline Bormann's I type & 18 & $0(0.0)$ & $18(33.3)$ & \\
\hline Bormann's II type & 13 & $0(0.0)$ & $13(24.1)$ & \\
\hline Bormann's I+II type & 11 & $0(0.0)$ & $11(20.4)$ & \\
\hline Bormann's III type & 9 & $0(0.0)$ & $9(16.7)$ & \\
\hline Bormann's IV type & 3 & $0(0.0)$ & $3(5.6)$ & \\
\hline Growth pattern & & & & $<0.001$ \\
\hline Expansive & 9 & $9(37.5)$ & $0(0.0)$ & \\
\hline Infiltrative & 69 & $15(62.5)$ & $54(100.0)$ & \\
\hline Lymphatic-vascular involvement & & & & $<0.001$ \\
\hline Absent & 20 & $15(62.5)$ & $5(9.3)$ & \\
\hline Present & 58 & $9(37.5)$ & $49(90.7)$ & \\
\hline Lymph node metastasis & & & & $<0.001$ \\
\hline Negative & 28 & $19(79.2)$ & $9(16.7)$ & \\
\hline Positive & 50 & $5(20.8)$ & $45(83.3)$ & \\
\hline Distant metastasis & & & & 0.001 \\
\hline pM0 & 54 & $23(95.8)$ & $31(57.4)$ & \\
\hline pM1 & 24 & $1(4.2)$ & $23(42.6)$ & \\
\hline
\end{tabular}

NET, neuroendocrine tumor; NEC, neuroendocrine carcinoma; MANEC, mixed adenoneuroendocrine carcinoma.

to the mucosa and submucosa. Soga (27) investigated 1,914 gastrointestinal carcinoids and hypothesized that the diameter of the tumor was closely associated with metastasis.
Gastrointestinal submucosa carcinoids exhibited a metastasic rate of $16.4 \%$ as a whole and minute carcinoids $(\leq 5 \mathrm{~mm})$ revealed a metastasic rate of $6.0 \%$ on average. In the present 
Table III. Association between clinicopathological factors and LN metastasis in 24 patients with submucosal NETs.

\begin{tabular}{|c|c|c|c|c|}
\hline \multirow[b]{2}{*}{ Parameter } & \multicolumn{3}{|c|}{ LN metastasis } & \multirow[b]{2}{*}{ P-value } \\
\hline & Patients, n & Negative, n (\%) & Positive, n (\%) & \\
\hline Gender & & & & 0.209 \\
\hline Male & 11 & $10(52.6)$ & $1(20.0)$ & \\
\hline Female & 13 & $9(47.4)$ & $4(80.0)$ & \\
\hline Age, years & & & & 0.774 \\
\hline$<30$ & 1 & $0(0.0)$ & $1(20.0)$ & \\
\hline $31-60$ & 14 & $13(68.4)$ & $1(20.0)$ & \\
\hline$>60$ & 9 & $6(31.6)$ & $3(60.0)$ & \\
\hline Tumor location & & & & 0.231 \\
\hline Esophagus & 2 & $2(10.5)$ & $0(0.0)$ & \\
\hline Duodenum & 2 & $2(10.5)$ & $0(0.0)$ & \\
\hline Stomach & 6 & $5(26.3)$ & $1(20.0)$ & \\
\hline Rectum & 14 & $10(52.6)$ & $4(80.0)$ & \\
\hline Histopathological classification & & & & $<0.001$ \\
\hline NET G1 & 20 & $19(100.0)$ & $1(20.0)$ & \\
\hline NET G2 & 3 & $0(0.0)$ & $3(60.0)$ & \\
\hline MANEC & 1 & $0(0.0)$ & $1(20.0)$ & \\
\hline Tumor diameter, mm & & & & 0.238 \\
\hline$\leq 5.0$ & 7 & $7(36.8)$ & $0(0.0)$ & \\
\hline $5.1-10.0$ & 13 & $9(47.4)$ & $4(80.0)$ & \\
\hline$>10.0$ & 4 & $3(15.8)$ & $1(20.0)$ & \\
\hline Macroscopic appearance & & & & 0.799 \\
\hline 0 -Is type & 18 & $15(78.9)$ & $3(60.0)$ & \\
\hline 0-Is+IIc type & 6 & $4(21.0)$ & $2(40.0)$ & \\
\hline Growth pattern & & & & 0.081 \\
\hline Expansive & 9 & $8(42.1)$ & $1(20.0)$ & \\
\hline Infiltrative & 15 & $11(57.9)$ & $4(80.0)$ & \\
\hline Envelope & & & & 0.607 \\
\hline Complete & 6 & $6(31.6)$ & $0(0.0)$ & \\
\hline Incomplete & 11 & $7(36.8)$ & $4(80.0)$ & \\
\hline $\mathrm{N} / \mathrm{A}$ & 7 & $6(31.6)$ & $1(20.0)$ & \\
\hline Lymphatic-vascular involvement & & & & 0.027 \\
\hline Absent & 15 & $14(73.7)$ & $1(20.0)$ & \\
\hline Present & 9 & $5(26.3)$ & $4(80.0)$ & \\
\hline Distant metastasis & & & & 0.049 \\
\hline pM0 & 23 & $19(100.0)$ & $4(80.0)$ & \\
\hline pM1 & 1 & $0(0.0)$ & $1(20.0)$ & \\
\hline
\end{tabular}

NET, neuroendocrine tumor; LN, lymph node; MANEC, mixed adenoneuroendocrine carcinoma; N/A, not applicable.

study, the majority of lesions measuring $<5.0 \mathrm{~mm}$ in diameter exhibited a slightly elevated macroscopic type and an expansive growth pattern without lymphatic-vascular involvement or distant metastasis. However, a high number of tumors measuring 5.1-10.0 $\mathrm{mm}$ in diameter exhibited an infiltrative growth pattern, lymphatic-vascular involvement and nodal metastasis. In the deeper invasion NETs group, all lesions measured $>10.0 \mathrm{~mm}$ and exhibited an infiltrative growth pattern. This indicated that at the initial stage of growth
( $\leq 5.0 \mathrm{~mm}$ in diameter), NETs exhibit an expanding growth pattern, and gradually develop an infiltrating growth pattern as the tumor increases in diameter $(>5.0 \mathrm{~mm})$. Subsequently, the tumor may invade through the submucosal layer, infiltrating the muscular and deeper layers. In addition, tumors may grow towards the mucosa in an elevated way, leading to mucosal erosions and ulcers (Borrmann's type II or III tumors). Thus, the present authors hypothesize that minute tumors with an expansive growth pattern present a relatively 
Table IV. Kaplan-Meier survival analysis of gastrointestinal NETs patients with regard to clinicopathological factors.

\begin{tabular}{|c|c|c|c|c|c|}
\hline Parameter & Patients, n & $\begin{array}{c}\text { Median overall } \\
\text { survival, months }\end{array}$ & $\begin{array}{c}\text { 3-year } \\
\text { survival rate, \% }\end{array}$ & $95 \% \mathrm{CI}$ & P-value \\
\hline Gender & & & & & 0.276 \\
\hline Male & 42 & 80.4 & - & $70.2-90.6$ & \\
\hline Female & 36 & 73.5 & - & $60.1-87.0$ & \\
\hline Age, years & & & & & 0.089 \\
\hline$<30$ & 6 & 61.8 & - & $36.6-87.1$ & \\
\hline $31-60$ & 33 & 88.9 & - & 79.4-98.4 & \\
\hline$>60$ & 39 & 66.2 & - & $54.1-78.4$ & \\
\hline Histopathological classification & & & & & $<0.001$ \\
\hline NET G1 & 20 & - & 100.0 & - & \\
\hline NET G2 & 29 & - & 100.0 & - & \\
\hline NEC & 9 & - & 44.4 & - & \\
\hline MANEC & 20 & - & 85.0 & - & \\
\hline Depth of invasion & & & & & 0.009 \\
\hline Submucosal & 24 & - & 100.0 & - & \\
\hline Deeper invasion & 54 & - & 83.3 & - & \\
\hline
\end{tabular}

${ }^{a}$ Overall and 3-year survival could not be calculated for parameters where patients remained alive (marked by the symbol, -). NET, neuroendocrine tumor; NEC, neuroendocrine carcinoma; MANEC, mixed adenoneuroendocrine carcinoma; CI, confidence interval.

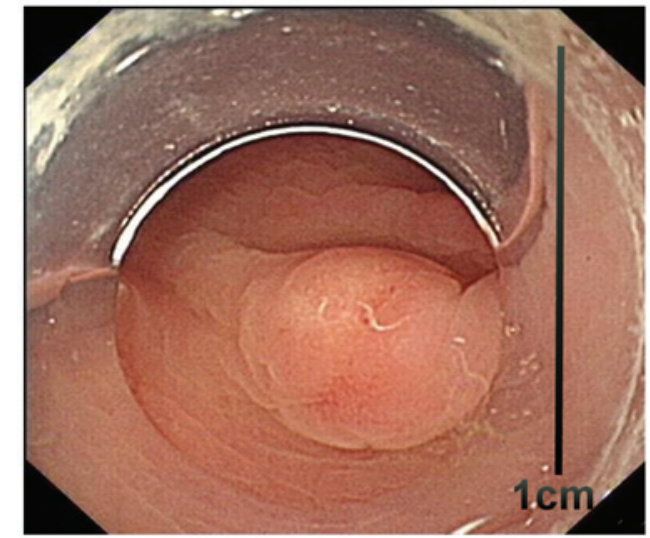

Figure 1. Endoscopic image of an early-stage gastrointestinal neuroendocrine tumor revealing a $0.6-\mathrm{cm}$ lesion of slightly lifted type.

inert stage of tumor development and therefore, such tumors are suitable for endoscopic therapy.

At present, surgery is considered the curative treatment for NETs with muscular layer or deeper infiltration, with or without LN metastasis $(13,28)$. Treatment for metastatic NETs includes, surgery, radiotherapy, chemotherapy and palliative care, depending on the individual case (28). If a tumor is limited to the mucosa or submucosa, it may be resected by surgery or by endoscopy using endoscopic mucosal resection or endoscopic submucosal dissection (15,16,29-31). Endoscopic resection is not only a curative method, but it also exhibits minimal trauma and a lower cost than other surgical procedures. For gastrointestinal epithelial tumors, if the tumor is diagnosed when limited to the mucosa or submucosa, $>50 \%$ tumors are curable, with a 5 -year survival rate of

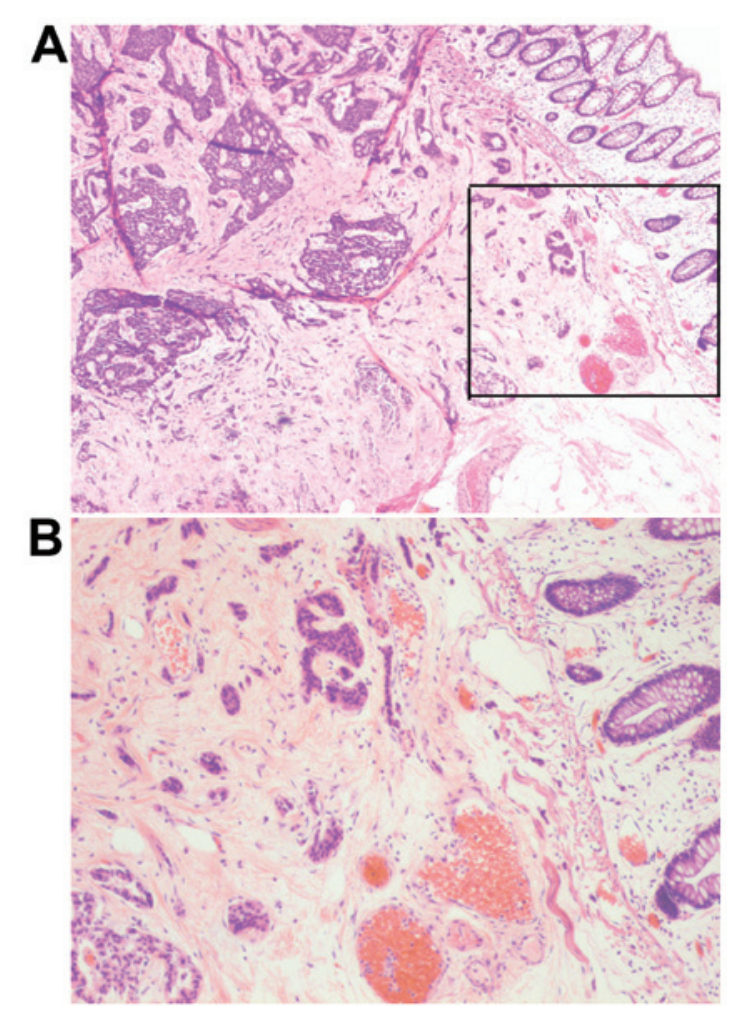

Figure 2. (A) Histological staining of an early-stage gastrointestinal neuroendocrine tumor measuring $0.6 \mathrm{~cm}$ in size and exhibiting an infiltrative growth pattern (hematoxylin and eosin staining; magnification, x40). (B) Magnified image of the panel delineated in part (A) (magnification, $\mathrm{x} 100$ ).

$80-90 \%$ (32). Generally, the indicators for endoscopic resection are as follows: The gastrointestinal NET is limited to the mucosa or submucosa; the diameter of the tumor is $<1.0 \mathrm{~cm}$; 
and there is no evidence of LN or distant metastasis $(15,16)$. Certain studies have reported endoscopic resection for tumors measuring $>1.0 \mathrm{~cm}$ in diameter (33-35). In the present study, 6 cases with lesions measuring $\leq 5.0 \mathrm{~mm}$ and 8 cases with 5.1-10.0-mm lesions in the submucosa were treated with tumor resection by endoscopy. A total of 5 patients underwent surgery following endoscopic resection for suspected positive margin or lymphatic-vascular involvement. No patients exhibited recurrence or metastasis during the follow-up period. These results indicated that surgery subsequent to endoscopic resection may achieve curable effects for patients with high risk factors. In the present study, submucosa NETs measuring 5.1-10.0 $\mathrm{mm}$ in diameter exhibited a high rate of lymphatic-vascular involvement $(46.2 \%)$ and LN metastasis (30.8\%), which suggests that the indicators for endoscopic resection of gastrointestinal NETs should be more stringent than that for early-stage gastrointestinal adenocarcinoma. For $\leq 5.0-\mathrm{mm}$ lesions of the slightly elevated type, endoscopic resection is a safe treatment method, whereas for gastrointestinal NETs of 5.1-10.0 $\mathrm{mm}$ in diameter, endoscopic resection must be performed with caution.

In the present study, overall prognosis was favorable. Survival time was significantly higher in early-stage and lower grade tumors compared with later-stage and higher grade tumors. For gastrointestinal carcinomas, once the tumor has progressed out of the range of being resectable, the patient is expected to possess a relatively poor prognosis. Thus, earlier diagnosis and improved systemic treatment for advanced disease is urgently required.

In conclusion, the present retrospective study revealed that gastrointestinal submucosal NETs regularly exhibit a slightly elevated macroscopic type, and are usually small low-grade tumors. These features may aid oncologists to diagnose gastrointestinal NETs at an early stage. Gastrointestinal NETs of $<5.0 \mathrm{~mm}$ in diameter with a slightly elevated macroscopic type may require endoscopic resection, while NETs measuring 5.1-10.0 mm in diameter must be considered carefully prior to attempting surgery, due to the potential risk of lymphatic-vascular involvement and LN metastasis.

\section{Acknowledgements}

The present study was supported by the National Natural Science Foundation of China (Beijing, China; grant nos. 81201640 and 81372302), Zhejiang Provincial Natural Science Foundation of China (Hangzhou, China; grant no. LY16H160026), the National Health Key Special Fund (Beijing, China; grant no. 200802112) and the Key Project of Zhejiang Province (Hangzhou, China; grant no. 2013C03044-5).

\section{References}

1. Modlin IM, Lye KD and Kidd M: A 5-decade analysis of 13,715 carcinoid tumors. Cancer 97: 934-959, 2003.

2. Pape UF, Berndt U, Müller-Nordhorn J, Böhmig M, Roll S, Koch M, Willich SN and Wiedenmann B: Prognostic factors of long-term outcome in gastroenteropancreatic neuroendocrine tumours. Endocr Relat Cancer 15: 1083-1097, 2008.

3. Hauso O, Gustafsson BI, Kidd M, Waldum HL, Drozdov I, Chan AK and Modlin IM: Neuroendocrine tumor epidemiology: Contrasting Norway and North America. Cancer 113: 2655-2664, 2008
4. Yao JC, Hassan M, Phan A, Dagohoy C, Leary C, Mares JE, Abdalla EK, Fleming JB, Vauthey JN, Rashid A and Evans DB: One hundred years after 'carcinoid': Epidemiology of and prognostic factors for neuroendocrine tumors in 35,825 cases in the United States. J Clin Oncol 26: 3063-3072, 2008.

5. Berge $\mathrm{T}$ and Linell F: Carcinoid tumours. Frequency in a defined population during a 12-year period. Acta Pathol Microbiol Scand A 84: 322-330, 1976.

6. Oberg K and Eriksson B: Endocrine tumours of the pancreas. Best Pract Res Clin Gastroenterol 19: 753-781, 2005.

7. Modlin IM, Oberg K, Chung DC, Jensen RT, de Herder WW, Thakker RV, Caplin M, Delle Fave G, Kaltsas GA, Krenning EP, et al: Gastroenteropancreatic neuroendocrine tumours. Lancet Oncol 9: 61-72, 2008.

8. Scherübl H, Cadiot G, Jensen RT, Rösch T, Stölzel U and Klöppel G: Neuroendocrine tumors of the stomach (gastric carcinoids) are on the rise: Small tumors, small problems? Endoscopy 42: 664-671, 2010.

9. Scherübl H: Rectal carcinoids are on the rise: Early detection by screening endoscopy. Endoscopy 41: 162-165, 2009.

10. Scherübl H, Jensen RT, Cadiot G, Stölzel U and Klöppel G: Neuroendocrine tumors of the small bowels are on the rise: Early aspects and management. World J Gastrointest Endose 2: 325-334, 2010

11. Strosberg J, Gardner N and Kvols L: Survival and prognostic factor analysis of 146 metastatic neuroendocrine tumors of the mid-gut. Neuroendocrinology 89: 471-476, 2009.

12. Zar N, Garmo H, Holmberg L, Rastad J and Hellman P. Long-term survival of patients with small intestinal carcinoid tumors. World J Surg 28: 1163-1168, 2004.

13. Scherübl H, Jensen RT, Cadiot G, Stölzel U and Klöppel G: Management of early gastrointestinal neuroendocrine neoplasms. World J Gastrointest Endosc 3: 133-139, 2011.

14. Kulke MH, Benson AB III, Bergsland E, Berlin JD, Blaszkowsky LS, Choti MA, Clark OH, Doherty GM, Eason J, Emerson L, et al: Neuroendocrine tumors. J Natl Compr Canc Netw 10: 724-764, 2012.

15. Zhou FR, Huang LY and Wu CR: Endoscopic mucosal resection for rectal carcinoids under micro-probe ultrasound guidance. World J Gastroenterol 19: 2555-2559, 2013.

16. Yamaguchi $N$, Isomoto $H$, Nishiyama $H$, Fukuda $E$, Ishii $H$ Nakamura T, Ohnita K, Hayashi T, Kohno S, Nakao K and Shikuwa S: Endoscopic submucosal dissection for rectal carcinoid tumors. Surg Endosc 24: 504-508, 2010.

17. Participants in the Paris Workshop: The Paris endoscopic classification of superficial neoplastic lesions: Esophagus, stomach, and colon: November 30 to December 1, 2002. Gastrointest Endosc 58 (Suppl 6): S3-S43, 2003.

18. Endoscopic Classification Review Group: Update on the Paris classification of superficial neoplastic lesions in the digestive tract. Endoscopy 37: 570-578, 2005.

19. Borrmann R: Tumors of the stomach and duodenum. In: Handbook of Special Pathological Anatomy and Histology: Digestive Tract. Borchardt H, Borrmann R, Christeller E, Dietrich A, Fischer W, Von Gierke E, Hauser G, Kaiserling C, Koch M, Koch W, et al (eds). Vol. 4. 1st edition. Springer Vienna, Berlin, p865, 1926 (In German).

20. Bosman FT, Carneiro F, Hruban RH and Theise ND (eds): WHO Classification of Tumours of the Digestive System. Vol 3. 4th edition. IARC Press, Lyon, 2010.

21. Pape UF, Jann H, Müller-Nordhorn J, Bockelbrink A, Berndt U, Willich SN, Koch M, Röcken C, Rindi G and Wiedenmann B: Prognostic relevance of a novel TNM classification system for upper gastroenteropancreatic neuroendocrine tumors. Cancer 113: 256-265, 2008.

22. Garcia-Carbonero R, Capdevila J, Crespo-Herrero G, Díaz-Pérez JA, Martínez Del Prado MP, Alonso Orduña V, Sevilla-García I, Villabona-Artero C, Beguiristain-Gómez A, Llanos-Muñoz M, et al: Incidence, patterns of care and prognostic factors for outcome of gastroenteropancreatic neuroendocrine tumors (GEP-NETs): Results from the National Cancer Registry of Spain (RGETNE). Ann Oncol 21: 1794-1803, 2010.

23. Cho MY, Sohn JH, Jin SY, Kim H, Jung ES, Kim MJ, Kim KM, Kim WH, Kim JM, Kang YK, et al; Gastrointestinal Pathology Study Group of Korean Society of Pathologists: Proposal for a standardized pathology report of gastroenteropancreatic neuroendocrine tumors: Prognostic significance of pathological parameters. Korean J Pathol 47: 227-237, 2013. 
24. Yoshino T, Shimoda T, Saito A, Nakanishi Y, Yusuke T, Shirasu T and Miura S: Macroscopic features and its clinical treatment of stomach type differentiated adenocarcinoma in early gastric cancer. Stomach and Intestines 34: 513-525, 1999 (In Japanese).

25. Japanese Gastric Cancer Association. Japanese gastric cancer treatment guidelines 2010 (ver. 3). Gastric cancer 14: 113-123, 2011.

26. Thomas RM, Baybick JH, Elsayed AM and Sobin LH: Gastric carcinoids. An immunohistochemical and clinicopathologic study of 104 patients. Cancer 73: 2053-2058, 1994.

27. Soga J: Early-stage carcinoids of the gastrointestinal tract: An analysis of 1,914 reported cases. Cancer 103: 1587-1595, 2005.

28. Ramage JK, Ahmed A, Ardill J, Bax N, Breen DJ, Caplin ME, Corrie P, Davar J, Davies AH, Lewington V, et al: Guidelines for the management of gastroenteropancreatic neuroendocrine (including carcinoid) tumours (NETs). Gut 61: 6-32, 2012.

29. Basuroy R, Srirajaskanthan R, Prachalias A, Quaglia A and Ramage JK: Review article: The investigation and management of gastric neuroendocrine tumours. Aliment Pharmacol Ther 39: 1071-1084, 2014

30. Heo J, Jeon SW, Jung MK, Kim SK, Shin GY, Park SM, Ahn SY, Yoon WK, Kim M and Kwon YH: A tailored approach for endoscopic treatment of small rectal neuroendocrine tumor. Surg Endosc 28: 2931-2938, 2014
31. Kwon YH, Jeon SW, Kim GH, Kim JI, Chung IK, Jee SR, Kim HU, Seo GS, Baik GH, Choi KD and Moon JS: Long-term follow up of endoscopic resection for type 3 gastric NET. World J Gastroenterol 19: 8703-8708, 2013

32. Sano T, Sasako M, Kinoshita T and Maruyama K: Recurrence of early gastric cancer. Follow-up of 1475 patients and review of the Japanese literature. Cancer 72: 3174-3178, 1993.

33. Sung HY, Kim SW, Kang WK, Kim SY, Jung CK, Cho YK, Park JM, Lee IS, Choi MG and Chung IS: Long-term prognosis of an endoscopically treated rectal neuroendocrine tumor: 10 -year experience in a single institution. Eur J Gastroenterol Hepatol 24: 978-983, 2012.

34. Yokoyama S, Takifuji K, Tani M, Kawai M, Naka T, Uchiyama $\mathrm{K}$ and Yamaue $\mathrm{H}$ : Endoscopic resection of duodenal bulb neuroendocrine tumor larger than $10 \mathrm{~mm}$ in diameter. BMC Gastroenterol 11: 67, 2011.

35. Baek IH: Endoscopic submucosal dissection or conventional endoscopic mucosal resection is an effective and safe treatment for rectal carcinoid tumors: A retrospective study. J Laparoendosc Adv Surg Tech A 20: 329-331, 2010. 